\title{
Über den Verfall paläobotanischer Forschung in den Ländern deutscher Zunge.
}

\author{
Von Dr. Bruno Kubart (Graz) ${ }^{1}$ ).
}

Vor einiger Zeit erhielt ich einen Sonderdruck von meinem Stockholmer Fachkollegen T. G. Halle : „Lower devonian plants from Röragen “ ${ }^{2}$ ). Wie gewöhnlich durchflog ich bei der Durchsicht der Arbeit auch die Literaturliste. Sie umfaßt volle drei Seiten und bietet das mir gewohnte Bild: Unter rund 110 angefübrten Arbeiten befinden sich 27 deutsch geschriebene, worin fünf von Skandinaviern in deutscher Sprache abgefaßte Arbeiten miteingerechnet sind, so daß also auf Autoren deutscher Zunge überbaupt nur 22 Arbeiten entfallen.

Dem war nicht immer so. Gerne wird in neuerer Zeit der „Materialmangel" als Ursache des fast völligen Darniederliegens paläobotanischer Forschung in den Zändern deutscher Zunge von den Deutschen selbst angegeben. So findet sich z. B. im Solms-Laubach-Nekrolog in den Ber. d. D. bot. G. rom Jahre 1915 aus der Feder eines deutseben Botanikers folgende Stelle: „In Deutschland existiert keine phytopaläontologische Schule und auch Solms hat keine solche begründet, obwohl er mebr wie jeder andere dazu imstande gewesen wäre, da er alle wichtigen Funde aus eigener Anschanung kannte und die Gesamtliteratur übersah. Unsele Jugend interessierte sich aber nicht für diese Wissenschaft; vermutlich, weil sie kaum Gelegenheit hat, Fossilien mit erhaltener Struktur zu sammein. In England und Frankreich, wo solche Materialien häufiger sind, da blüht auch diese Wịsenschaft ganz anders als bei uns. Namentlich in Encland ist denn auch Solms sehr anerkannt worden." Ganz im gleichen Sinne schreibt W. Nieburg ${ }^{3}$ ) neuestens in der Naturwiss. Wochenschrift.

Besteht denn nun wirklich "Materialmangel"? Ein Paläobotaniker wird dies wohl kaum behaupten! Liegen doch in unseren Sammlungen Kasten und Laden voll Material, das teils unbearbeitet ist oder dringendst einer Neubearbeitung bedarf; in der freien Natur kann weiters reichlich neues gesammelt werden und auch die weite Welt wird uns wieder offen stehen, um von dorten fremdartiges Material beschaffen zu können,

1) Mit voller Absicht wurden in die folgenden Ausführungen so viel als möglich $\ddot{A} u ß e r u n g e n$ anderer Autoren eingefügt.

2) Halle T. G.: "Lower devonian plants from Röragen“, K. Svenska Vet. Handlingar, 1916.

3) N ieburg W.: „Neue Wege der phylogenetischen Pflanzenanatomie", Naturw. Wochenschrift, Bd. 33 (1918), S. 105 u. f. 
an dem wir dann im Vergleiche mit unserem heimischen Materiale unser Urteil schärfen können. Müssen es denn aber auch gerade immer nur Fossilien mit "erhaltener Struktur" sein, mit denen man arbeiten möchte, weil es eben jetzt so "Mode" ist? Wir wissen doch heute zur Genüge, daß auch aus Abdruckfossilien, wenn nur ein kohliger Belag erhalten geblieben ist, direkt staunenswerte Ergebnisse gewonnen werden können. Man lese nur einmal in den Arbeiten des Schweden Nathorst nach, der diese Arbeitsmethode besonders ausgebildet und verwertet hat. Aber auch an Fossilien mit erhaltener Struktur gibt es bei uns. keinen Mangel. So sind z. B. die fossilen Hölzer, die sich bei uns finden, vielfach überhaupt nicht oder keineswegs genau holzanatomisch bearbeitet. Und selbst das jetzt so viel genannte Material der Engländer für die Untersuchungen eines Williamson, Scott und Oliver, u. a, die sogenannten coal-balls, das sind die "Torfdolomite" der deutschen Bergleute und die „Pflauzensphaerosiderite" der Österreicher, ist bei uns. in Mengen vorhanden, nur daß sich eben niemand darum gekümmert hat ${ }^{1}$ ). Die Annahme, daG das Studium unserer Torfdolomite vielleicht keine besonderen Resultate mehr bieten würde, da die Engländer schon die eigenen so ausgiebig untersucht haben, eine Meinung, die vielleicht. tatsächlich auch Solms hatte, kann ich nach meinen eigenen Erfahrungen absolut nicht teilen. Vor allem habeu ja die Engländer selbst noch nicht einmal eine durchgreifende Erforschung ihrer heimischen Torfdolomite beendet, denn diese Arbeiten sind mühevoll und erfordern daher, wollen sie gründlich gemacht werden, lange Zeit. Wir müssen aber vollauf anerkennen, $d a ß$ in England für paläobotanische Arbeiten großes Interesse besteht; so hat sich dort sogar ein Grubenbesitzer entschlossen, einen Schacht, der zwar nicht ergiebig war, dessen Flötze aber besonders reich an den wissensehaftlich so wertvollen Torfdolomiten sind, wieder in Betrieb zu nehmen, um vor allem genaue Studien über das Vorkommen dieser Materialien in der Grube selbst zu ermöglichen. Hiebei mu@ aber noch berücksichtigt werden, daß auch sonst in englischen Gruben Torfdolomite viel reichlicher vorkommen als bei uns. Auch ein allfälliger Einwand, die Güte des bei uns verfügbaren Materiales lasse viel zu wünschen übrig, kann nicht aufrecht erbalten werden. So ist, um nur ein Beispiel herauszugreifen, der Erhaltungszustand der in den österreichischen Torfdolomiten eingeschlossenen Pflauzen vielfach sogar ein besserer, als jener des englischen Materiales. Im übrigen kann hier als Zeuge die berufenste englische Stimme selbst, der Paläobotaniker Scott, angeführt werden, der am Schlusse seiner "Studies in fossil botany", 2. Bd., 1909, schreibt: "As this book goes to press, I have

1) siebe z. B.: Kubart B., Pflanzenversteinerungen enthaltende Knollen...", Sitzb. d. Ak. d. Wiss. in Wien, m.-n. Klasse, Bd. 107, Abt. 1, 1908. 
received from Dr.K.... sections from the magnificently preserved nodules of the Moravian coal-measures, a field hitherto unworked from a structural point of view"1). Das mir bisher zu vergleichenden Arbeiten zur Verfügung stehende reichsdeutsche Torfdolomitmaterial birgt allerdings nicht so günstig erhaltene Pflanzenreste, doch glaube ich bestimmt, was übrigens auch Felix sagt, dafs reichlicheres deutsches Material desgleichen sehr gute Präparate liefern wird. Reichliches Material kann aber beschafft werden, es gilt nur dasselbe einzusammeln, denn aus mehreren deutschen Gruben sind heute bereits Torfdolomite bekannt und gesteigertes Interesse wird sicherlich auch neue Fundstellen aufdecken helfen. Unrecht tut man aber auch unseren Hörern, wenn man ihnen Mangel an Interesse vorwirft. Finden sie denn an unseren deutschen Hochschulen auch Gelegenheit, phytopaläontologische Vorlesungen oder Übungen zu besuchen oder gar selbst eine phytopaläontologische Arbeit auszuführen? Ich kann das Gegenteil von unseren Hörern behaupten und möchte auch aus eigener Erfahrung das große Interesse der breiten Masse des Volḳes für Vorträge über die Pflanzen der Vorwelt hervorheben.

Die wirklichen Ursachen für diesen Tiefstand paläobotanischer Forschung in unserer Heimat - man kann eigentlich sagen in ganz Mitteleuropa ${ }^{2}$ ) - scheinen wohl ganz wo anders zu liegen, und schon ein kurzer historischer Rückblick weist deutlich auf sie hin, indem er zugleich auch andeutet, wie diesem Tiefstande abzuhelfen wäre.

Seit der Mitte des vorigen Jahrhunderts wurde bei uns die gesamte Paläontologie fast ausschließlich Domäne der Geologie (Jaekel), was aber für die Paläontologie zum Unglück wurde ${ }^{3}$ ). Denn für den

1) Anderweitige dienstliche Verplichtungen behinderten leider bisher die entsprechende Auswertung dieses wirklich herrlichen Materiales.

2) Knapp vor Beginn des Kampfes um unsere Zukunft veröffentlichte der ordentliche Professor der Botanik an der Prager tschechischen Universität J. Velenovský einen mit diesen Ausführungen völlig gleichsinnigen Artikel über den Betrieb der Phytopaläontologie bei den Tschechen (Budoucí úkoly fỵtopaleontologie v Čechách im "Čsopis Musea království Českého", 1914 = Die zúkünftigen Aufgaben der Phytopaläontologie in Böhmen in, Zeitschrift des Museums des Königreiches Böhmen"). Bei Anführung dieser Arbeit muß ich jedoch bemerken, daß ich. nur den Ausführungen Velenorskys über Stellung, Bedeutung und Pflege der Phytopaläontologie beipflichte, seine eingeflochtenen, im höchsten Maße gehässigen Anwürfe gegen die deutsche Wissenschaft aber selbstredend mit aller Entschiedenheit zurückweise.

3) Auffallenderweise verfiel auch die Pflege der Paläobotanik in Österreich vollends, obwobl sich gerade hier schon frühzeitig Botaniker wie Cord a und Graf Sternberg in Prag, vor allem aber Unger in Graz, von dem u. a. auch die ersten Vegetationsbilder früherer Erdperioden stammen, um die wis sen-. sehaftliche Begründung der Paläobotanik historisehe Verdienste erworben hatten. 
Geologen, der doch ganz andere Aufgaben vor sich hat, mußte die gesamte Paläontologie, also Phytopaläontologie (Paläobotanik) und Zoopaläontologie, unbedingt nur zum Range einer Hilfswissenschaft herabsinken. Nur die "Leitfossilien" sind für den Geologen von Bedeutung; diese stellen jedoch bloß einen kleinen Bruchteil aller Fossilien dar. Die geologische Bedeutung der vielen $\mathrm{Nichtleitfossilien} \mathrm{ist} \mathrm{aber}$ eine minimale oder völlig nichtige und diese sind dem Geologen sohin auch gleichgiltig. Für den Botaniker und Zoologen haben jedoch alle Fossilien Wert und Bedeutung, und auch nur dieser kann sie dank seiner Vorschulung entsprechend auswerten. „Es gibt allerdings auch heute noch viele Nicht-Zoologen und Nicht-Botaniker, die sich mit der Beschreibung von Fossilien beschäftigen, insbesondere soferne es sich um ausgestorbene niedere Tiere handelt. Arbeiten von systematischem Werte setzen aber selbstredend zoologische, bzw. botanische Fachkenntnisse voraus" $\left(\mathrm{Nathorst)}{ }^{1}\right)$. Diese Erkenntnis mag heute allerdings noch nicht bei allen deutschen Geologen vorhanden sein, sie kommt aber in letzter Zeit unzweifelhaft immer mehr und mehr zur Geltung. So stehen z. B. Jaekel${ }^{2}$ ) und $\mathrm{Branca}^{3}$ ) auf diesem Standpunkte und Branca schrieb hierüber 1910: „Wir heutigen geologischen Paläontologen haben zwar ja alle, der eine otwas mehr, der andere etwas weniger, Zoologie gehört und etwas praktisch gearbeitet; und wir haben dann im Laufe unseres wissenschaftlichen Lebens, je nach unserer Arbeitsrichtung, unsere zoologischen Kenntnisse mehr oder weniger weiter zu vertiefen gesucht. Indessen, das ist doch offenbar nur eine völlig mangelhafte, minderwertige, der Wissenschaft dann nicht würdige Vorbereitung, wenn man die Paläontologie ....... als eine rein zoologische Wissenschaft behandeln will." Das gleiche gilt natürlich auch für die Phytopaläontologie als botanische Wissenschaft. Selbstredend mus aber der Zoo- und Phytopaläontologe so weit geschult sein. daß er die Angaben, welche die Geologen ihm über das Alter der betreffenden Schichten machen, mit eigenem Urteil verstehen kanu. Aber wesentlich weiter braucht für ihn das Studium der Geologie nicht mehr zu gehen (Branca).

Der nichthotanische Betrieb der Paläobotanik mußte selbstredend zum völligen Verfall dieser Wissenscbaft bei uns führen. Der deutsche Botauiker Schenk, der selbst mustergiltig paläobotanisch arbeitete, hat

1) Nathorst: „Über paläobotanische Museen“, Engle r's botan. Jahrbuch, Bd. 42, 1909.

2) J a ekel: „Über Geologie und Paläontologie an den deutschen Hochschulen”, Naturw. Wochenschrift, 1910.

3) Branca: „Über die Abtrennung der Paläontologie von der Geologie", Naturw. Wochenschrift, 1910. 
dies im Vorwort seiner Bearbeitung der fossilen Planzen in Zittels Handbuch der Paläontologie mehr als deutlich zum Ausdruck gebracht. Dies war 1890. Schenk wollte Gutes stiften, aber das Gegenteil trat ein und die Paläobotanik kam bei den deutschen Botanikern noch mehr in Verruf. Sicher wäre es besser gewesen, nun selbst erst recht zur Sache zu sehen, um sich doch durch eigene Beobachtung zu überzeugen, ob denn da keine Aussicht auf Erfolg und Fortschritt bestehe. Amerikaner, Engländer, Franzosen und Schweden ${ }^{1}$ ) ließen sich dadurch nicht beirren, arbeiteten rüstig weiter und heute feiert deutsche Botanik die großartigen Ergebnisse der Paläobotanik, die Ausländer in der letzten Zeit nach jahrelanger, mühevollor Arbeit errungen haben. Wir stehen eigentlich beschämt da, denn einen großen Teil der Erfolge hätten wir selbst erzielen können, wenn wir das bei uns vorbandene Material ausgewertet $\mathrm{b}$ äten und hiezu die nötigen Vorbedingungen (Behelfe und Arbeitsgelegenheiten) geboten hätten!

\title{
Vorarbeiten zu einer Flechtenflora Dalmatiens.
}

\author{
Von Dr. A. Zahlbruckner (Wien).
}

VII.

(Fortsetzung ${ }^{2}$ ).

\section{Stictaoeae.}

Lobaria pulmonaria (L.) Hoffm. - Vorarb. Nr. 248.

Arbe: Dundowald, ca. $80 \mathrm{~m}$, an Eichen, steril (Baumgartner); West-Curzola: Gipfel des Kom bei Smokvica, ca. $500 \mathrm{~m}$ (Baumgartner); Meleda: Anhöben über Porto Soline, ca. $300 \mathrm{~m}$, im Macchiengebüsch̀ und über Knežovo polje bei Govadjari, $100-150 \mathrm{~m}$ (Baumgartner).

1) Bereits im Jahre 1884 gründeten Regierung und Reichsrat von Sehweden eine eigene paläobotanische Abteilung an den Sammiungen der schwedischen Akademie der Wissenschaften in Stockholm und 1915 finden wir Botanik und Paläobotanik gemeinsam in einem der neuen Gebäude der schwedischen Akademie auferhalb Stoekholms untergebracht. Als echte Schwesterdisziplinen teilen sich hier Botanik und Paläobotanik in die beiden Hälften des 67 m langen Hauses.

2) Siehe: Österr. botan. Zeitschrift, Band LXVIII, 1919, Heft 1/4: S. 60-77; Heft 5/7: S. 148-165. 